\title{
Medial temporal lobe atrophy and memory dysfunction as predictors for dementia in subjects with mild cognitive impairment
}

Citation for published version (APA):

Visser, P. J., Scheltens, P., Verhey, F. R. J., Schmand, B., Launer, L. J., Jolles, J., \& Jonker, C. (1999). Medial temporal lobe atrophy and memory dysfunction as predictors for dementia in subjects with mild cognitive impairment. Journal of Neurology, 246(6), 477-485. https://doi.org/10.1007/s004150050387

Document status and date:

Published: 01/01/1999

DOI:

10.1007/s004150050387

Document Version:

Publisher's PDF, also known as Version of record

Document license:

Unspecified

Please check the document version of this publication:

- A submitted manuscript is the version of the article upon submission and before peer-review. There can be important differences between the submitted version and the official published version of record.

People interested in the research are advised to contact the author for the final version of the publication, or visit the DOI to the publisher's website.

- The final author version and the galley proof are versions of the publication after peer review.

- The final published version features the final layout of the paper including the volume, issue and page numbers.

Link to publication

\footnotetext{
General rights rights.

- You may freely distribute the URL identifying the publication in the public portal. please follow below link for the End User Agreement:

www.umlib.nl/taverne-license

Take down policy

If you believe that this document breaches copyright please contact us at:

repository@maastrichtuniversity.nl

providing details and we will investigate your claim.
}

Copyright and moral rights for the publications made accessible in the public portal are retained by the authors and/or other copyright owners and it is a condition of accessing publications that users recognise and abide by the legal requirements associated with these

- Users may download and print one copy of any publication from the public portal for the purpose of private study or research.

- You may not further distribute the material or use it for any profit-making activity or commercial gain

If the publication is distributed under the terms of Article $25 \mathrm{fa}$ of the Dutch Copyright Act, indicated by the "Taverne" license above, 
Pieter Jelle Visser Philip Scheltens Frans R. J. Verhey Ben Schmand Leonore J. Launer Jellemer Jolles Cees Jonker

\section{Medial temporal lobe atrophy and memory dysfunction as predictors for dementia in subjects with mild cognitive impairment}

Received: 18 June 1998

Received in revised form: 28 September 1998 Accepted: 4 November 1998

P. J. Visser · F. R. J. Verhey · J. Jolles

Department of Psychiatry

and Neuropsychology

Institute of Brain and Behavior

University of Maastricht

Maastricht, The Netherlands

P. Scheltens ( $\triangle)$

Department of Neurology

Academisch Ziekenhuis VU

P.O. Box 7057

NL-1007 MB Amsterdam

The Netherlands

e-mail: p.scheltens@azvu.nl

Tel.: +31-20-4444444

Fax: +31-20-4440197

L. Launer

RIVM, Bilthoven, The Netherlands

C. Jonker

Department of Psychiatry

Vrije Universiteit

Amsterdam, The Netherlands

B. Schmand

Department of Neurology

Academic Medical Center

University of Amsterdam

Amsterdam, The Netherlands
Abstract To determine whether the medial temporal lobe is atrophic in subjects with mild cognitive impairment, and whether atrophy of this structure is a better predictor of dementia than memory dysfunction. Forty-five noninstitutionalized subjects aged 65-85 years were randomly selected from a population based study to obtain a sample with Alzheimer's disease (AD; $n=7)$, and a clinically nondemented sample $(n=38)$. Twenty of the latter subjects displayed some cognitive impairment and fulfilled CAMDEX criteria for "minimal dementia." Coronal T1-weighted magnetic resonance imaging was used to visualize the medial temporal lobe. The volume of the parahippocampal gyrus and hippocampus was measured, and medial temporal lobe atrophy was assessed qualitatively. The memory subscore from the CAMCOG was used as a measure of memory functioning. The follow-up period was 3 years. Nine subjects who were diagnosed as being minimally demented at baseline met the criteria for AD during follow-up. At baseline the volume of the parahippocampal gyrus of these subjects was smaller than that of the other subjects with minimal dementia. The memory score was the best predictor of clinical outcome. All medial temporal lobe measures increased the accuracy of prediction compared with only the memory score, by reducing the number of false-negative classifications of dementia. Severe medial temporal lobe atrophy is present even in some subjects with mild cognitive impairment and is an indicator of subsequent $\mathrm{AD}$. The absence of medial temporal lobe atrophy, however, does not exclude the development of dementia. In the majority of subjects memory impairment was a better predictor of dementia than atrophy of the medial temporal lobe. The combination of the two increased predictive accuracy. Nondemented subjects with severe atrophy of the medial temporal lobe could be enrolled in drug trials aimed at slowing the progression of $\mathrm{AD}$.

Key words Hippocampus . Alzheimers disease · Follow-up studies $\cdot$ Memory disorders . Cognitive impairment no dementia

\section{Introduction}

Alzheimer's disease (AD) is the most common type of dementia. The diagnosis of AD requires cognitive impairment, including memory dysfunction that is severe enough to interfere with activities of daily living [25].
Cognitive symptoms and brain abnormalities, however, are present many years before a clinical diagnosis of $\mathrm{AD}$ can be made. This preclinical phase of $\mathrm{AD}$ is the subject of intensive investigation because earlier diagnosis of $\mathrm{AD}$ may allow drug therapy to be started earlier, which may improve the clinical response. 
The earliest neuropathological changes occur in the medial temporal lobe [3], which includes the hippocampus and parahippocampal gyrus. Atrophy of these structures can be detected by magnetic resonance imaging (MRI) in an early stage of $\mathrm{AD}[4,12,15,18,19,23,26$, $32,33]$. Because the medial temporal lobe plays an important role in the storage of new information [29, 35], this atrophy may explain why memory dysfunction is an early symptom of $\mathrm{AD}[27,36]$. Consistent with this, subjects with memory impairment who do not meet the criteria of dementia have an increased risk of subsequent $\mathrm{AD}$ $[2,9,24,37]$. In the same way atrophy of the hippocampus or parahippocampal gyrus increases the risk for subsequent $\mathrm{AD}$ in elderly nondemented individuals [5, 17], and in asymptomatic individuals at risk for autosomal dominant $\mathrm{AD}[11]$.

The present study investigated whether atrophy of the medial temporal lobe is present in mildly impaired subjects who later become demented. To obtain insight into the relationship between memory function and medial temporal lobe atrophy we compared the predictive value for dementia of medial temporal lobe atrophy with that of memory performance alone and the combination of the two. We also investigated the correlation between brain volumes at baseline and cognitive scores at baseline and the decline in cognitive scores at follow-up. In this way we investigated the relationship between medial temporal lobe atrophy and cognitive decline as a continuous variable rather than as a dichotomous one.

To exclude the possibility that medial temporal lobe atrophy is a reflection of generalized atrophy of the brain we also measured the volume of the remaining part of the temporal lobe. Further, to obtain reference values for cognitive function and brain volumes in $\mathrm{AD}$, we included mildly to moderately demented AD patients. These subjects did not take part in the follow-up study.

\section{Materials and methods}

Subjects

We examined participants in the Amsterdam Study of the Elderly (AMSTEL), a two-stage population-based study of mental functioning in noninstitutionalized persons aged 65-85 years living in Amsterdam, The Netherlands [20, 34]. From the 4051 members of the baseline cohort, 787 individuals, randomly selected by age (5-year strata, 65-69 to 80-84 years) and Mini-Mental State Examination score (MMSE) [10] (MMSE scores 21, 22-26, and $>27$ ) were asked to participate in a 3 -year follow-up study of cognitive function. Of these, 511 individuals $(65 \%)$ agreed to participate. For the neuroimaging study we randomly selected from the subjects of the follow-up study a subsample with a range of cognitive functions to include clinically normal individuals and mildly to moderately demented individuals [21]. Of the 73 individuals classified as suffering from minimal dementia (see below), 33 were asked to participate and $28(84 \%)$ agreed. As far as possible, a demented individual and a normal individual from the same 5-year age stratum and MMSE stratum were selected and asked to participate: $19(70 \%)$ of the 27 normal individuals agreed to participate and $16(76 \%)$ of the 21 demented individuals agreed. Thus, the sample overrepresented the group with minimal dementia. Among those with dementia severity there was no significant difference between those agreeing to participate and those who did not with respect to the CAMCOG score for global cognitive function and its subscale measuring memory function. All subjects gave their informed consent prior to inclusion in the neuroimaging study. Subjects with a diagnosis of depression or dementia due to causes other than $\mathrm{AD}$ were excluded $(n=9)$. Three of the resulting 54 scans could not be used because of movement artifacts and six because the scans had not been completely stored on magnetic tape. Of the nine subjects with missing MRI, eight had minimal dementia subjects and one had AD. Thus, the scans for 45 subjects were available: 38 nondemented subjects at baseline (20 normal subjects and 18 subjects with minimal dementia) and 7 demented subjects at baseline.

\section{Baseline assessment and clinical diagnosis}

Each subject was assessed for dementia by means of an examination conducted at home by a research nurse and a physician. The assessment included a questionnaire, cognitive tests, and a clinical examination with the validated Dutch version of the CAMDEX protocol $[6,30]$. The cognitive section of the CAMDEX, the CAMCOG, includes sections for testing memory, praxis, calculation, language, attention, concentration, abstract thinking, and orientation, and has a maximum score of 107. An informant interview was administered to the closest relative or caregiver. The diagnoses of dementia and AD were made according to the DSM-IIIR [1], and NINCDS-ADRDA criteria [25], respectively. The diagnosis of minimal dementia was made when the DSM-IIIR criteria of dementia were not met, but based on an overall clinical impression, there was limited and variable impairment in cognitive and social functioning such as difficulty with learning and recalling events, a tendency to misplace possessions, and minor errors in orientation. Similar entities are "questionable dementia" or a score of 0.5 on the clinical dementia rating scale [14], and "mild cognitive impairment" or a score of 3 on the global deterioration scale [28].

\section{Cognitive measures}

We used the CAMCOG total score, the memory subscore of the CAMCOG, and the MMSE. To calculate the change in these scores during follow-up we calculated the net change per year of follow-up. Several subjects who developed AD during the study dropped out before the third follow-up assessment; therefore we calculated an adjusted decline score. This score was based on the assumption that once $\mathrm{AD}$ was diagnosed at follow-up, the course of cognitive decline would be the same as that in the AD subjects at baseline. For example, in subjects with only a 1-year follow-up we added the average decline among the AD patients at baseline over 2 years and divided the total decline by three.

\section{Follow-up}

Subjects were reexamined annually for 3 years according to the CAMDEX protocol in order to assess the clinical outcome (normal, minimally demented, demented) and the changes in cognitive functioning. Subjects who left the study before the third assessment but after the diagnosis of $\mathrm{AD}$ was made were included in the analysis.

MRI acquisition and morphometric analyses

MRI was performed on a Teslacon II (Technicare, Solon, Ohio) operating at $0.6 \mathrm{~T}$, according to a standard protocol reported previ- 
Fig. 1 The hippocampus (green), the parahippocampus gyrus (blue), and the lateral part of the temporal lobe (yellow), at the level of the body of the hippocampus (a), and the head of the hippocampus (b)
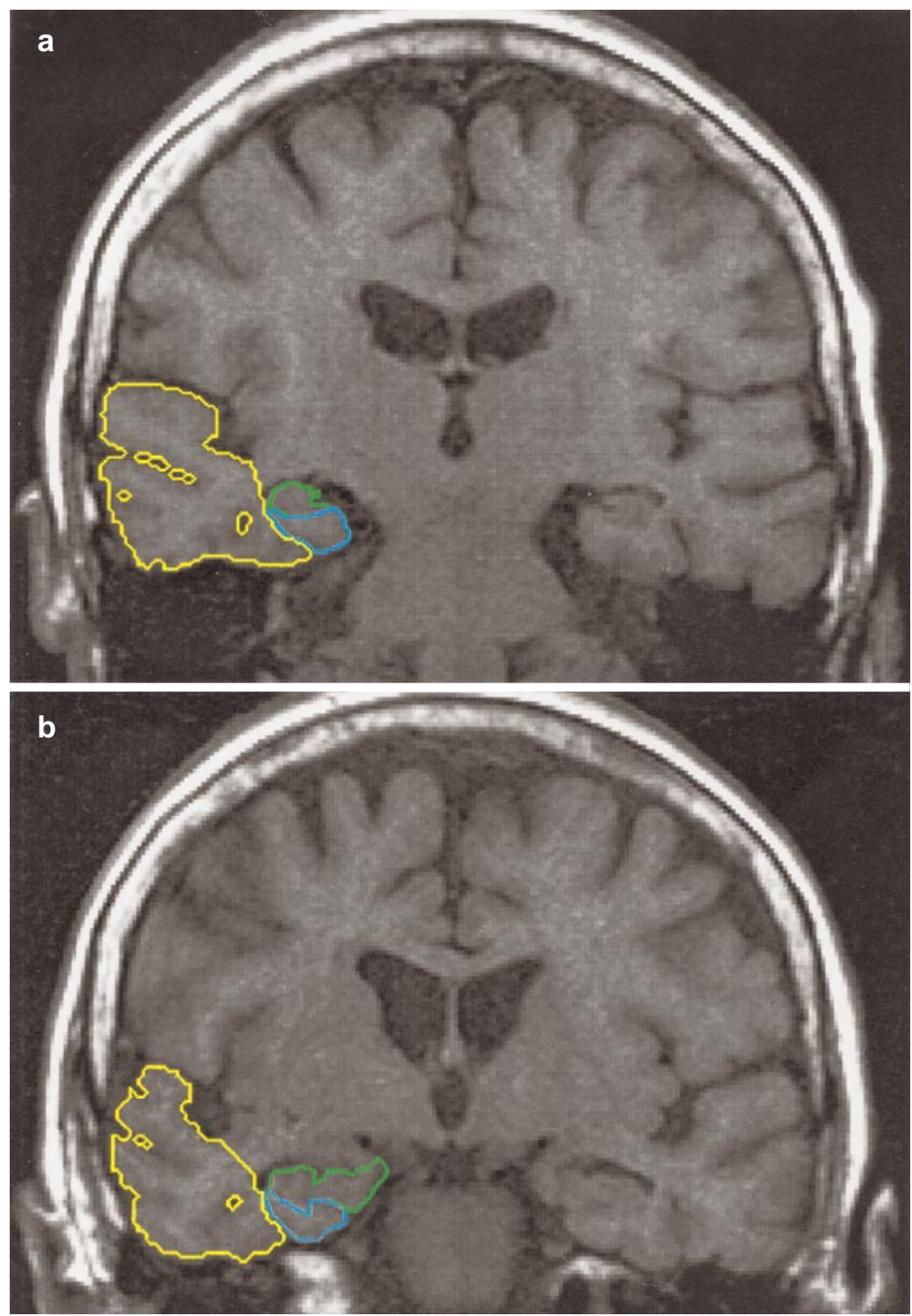

ously [33]. For this study we used six T1-weighted (TR $300 \mathrm{~ms}$, TE $22 \mathrm{~ms}$ ) coronal slices parallel to the brainstem axis and perpendicular to the hippocampal axis, planned from a midsagittal scout image. Slice thickness was $5 \mathrm{~mm}$ with an interslice gap of $1 \mathrm{~mm}$ and an in-plane resolution of $0.8 \times 1.0 \mathrm{~mm}$.

We selected four consecutive slices of which the first and second were made through the head of the hippocampus and the third and the fourth through the body of the hippocampus (Fig. 1). Volumetry was carried out on a SUN workstation with software developed in-house. The hippocampus, parahippocampal gyrus, and intracranial area, as a measure of the intracranial volume, were outlined by hand. A seed function was used for the temporal lobe. The volume of the hippocampus included the hippocampus proper, dentate gyrus, alveus, and subicilum. On the first slice the hippocampus was disconnected from the amygdala with a straight line through the lowest point of the connection between these two structures. The collateral sulcus was taken as the lateral border of the parahippocampal gyrus. The temporal lobe was disconnected from the rest of the brain at the temporal stem: a straight line was drawn from the deepest point of the fissura circularis to the upperlateral border of the lateral ventricle. For the intracranial area the outline of the inner table was followed. Below the temporal lobe either the outer border of the temporal gyri or the tentorium cerebellum was followed, and from the medial part of the temporal lobe a straight line was drawn to the bottom of the third ventricle. The volumes of the hippocampus and parahippocampal gyrus were substracted from the volume of the total temporal lobe to give the volume of the lateral temporal lobe. The volumes of the left and right sides were summed because the volumes of the two sides were not statistically significantly different from each other. To correct for individual differences in the volume of the intracranial area the brain volumes were adjusted (see below) and these adjusted values were used for all analyses. All measurements were carried out by one rater who was blinded to the subjects' age, diagnosis, and sex. The average difference between the first and second measurement of the brain structures on ten scans was $-0.08 \pm$ $0.29 \mathrm{~cm}^{3}$ for the parahippocampal gyrus, $-0.07 \pm 0.20 \mathrm{~cm}^{3}$ for the hippocampus, $0.14 \pm 1.1 \mathrm{~cm}^{3}$ for the lateral temporal lobe, and $-1.9 \pm 1.4 \mathrm{~cm}^{3}$ for the intracranial area.

Medial temporal lobe atrophy was assessed visually by a neurologist and radiologist in conference, who were blinded to the subjects' age, diagnosis, and sex. Scores ranged from 0 (no atro- 
phy) to 4 (severe atrophy). The rating scale is based upon a visual estimation of the volume of the medial temporal lobe, including the hippocampus proper, dentate gyrus, subicilum, and parahippocampal gyrus, and the volume of the surrounding CSF spaces, in particular the temporal horn of the lateral ventricle and the choroid fissure, on both sides. In cases of severe asymmetry the score of the more affected side was used. Results of the visual method of scoring are correlated well with linear and volumetric measurements and have an high intrarater reliability $(\kappa=0.70)[31,33,38]$.

Statistical analysis

The data were analyzed using SPSS for Macintosh 4.0. Categorical data were analyzed by the $\chi^{2}$ test. The $\chi^{2}$ test for trend was used to analyze medial temporal lobe atrophy scores. Group comparisons of continuous data listed in Table 1 were analyzed by using analysis of variance and corrected for multiple testing according to Bonferroni. Group comparisons of continuous data listed in Table 2 were analyzed by using the Mann-Whitney test. Linear regression was used to correct the brain volumes for differences in intracranial area, as described by Jack et al. [16]. Regression coefficients for this correction were derived from the regression analysis of the volume of the brain structures on the intracranial area of the nondemented subjects with completed follow-up and no evidence of cognitive decline. Logistic regression was used to assess the predictive value of memory score, parahippocampal gyrus, hippocampus, medial temporal lobe atrophy score, and the combination of memory score and the brain measures for clinical outcome at follow-up (demented vs nondemented). This analysis was performed with a combined sample of normal subjects and subjects with minimal dementia at baseline.

To assess whether the model improved after addition of one of the brain measures to the memory score, the change in -2 log likelihood ( $-2 \mathrm{LL})$ was tested. In the logistic regression analysis the memory score was corrected for age and education. This correction was based on the linear regression of these variables on the baseline memory score of a sample of 188 subjects from the AMSTEL study without diagnosis of dementia after a 3-year follow- up. The same method was used as for the correction of differences in the volume of the intracranial area (see above). The correlation between brain volume and cognitive score at baseline was calculated by linear regression with age and education as covariate, using data for the combined sample of normal subjects and minimally demented subjects. The correlation between brain volumes at baseline and change in cognitive scores during follow-up was calculated by linear regression with age as covariate. Since nine comparisons were made for the correlation between brain volumes and baseline cognitive scores or rate of cognitive decline, we adjusted for multiple testing according to Bonferroni and considered $P$ values lower than 0.0056 as significant.

\section{Results}

\section{Baseline characteristics}

Table 1 lists the baseline characteristics of the normal subjects, those with minimal dementia, and those with $\mathrm{AD}$ at baseline. Demographic variables were comparable between the groups. As expected, cognitive impairment was severe in the subjects with $\mathrm{AD}$ and mild in those with minimal dementia. Brain structures were smaller in the AD group than in the normal group, but this difference was only statistically significant for the parahippocampal gyrus. The volumes of the hippocampus and parahippocampal gyrus in the minimal dementia group were between those of the normal subjects and those with AD.

\section{Outcome at follow-up}

Nine subjects were lost before the first follow-up assessment and two between the second and the third because
Table 1 Demographic characteristics, cognitive scores and brain volumes at baseline

\footnotetext{
$* P<0.05$ vs normal group, $* * P<0.05$ vs minimal dementia group
}

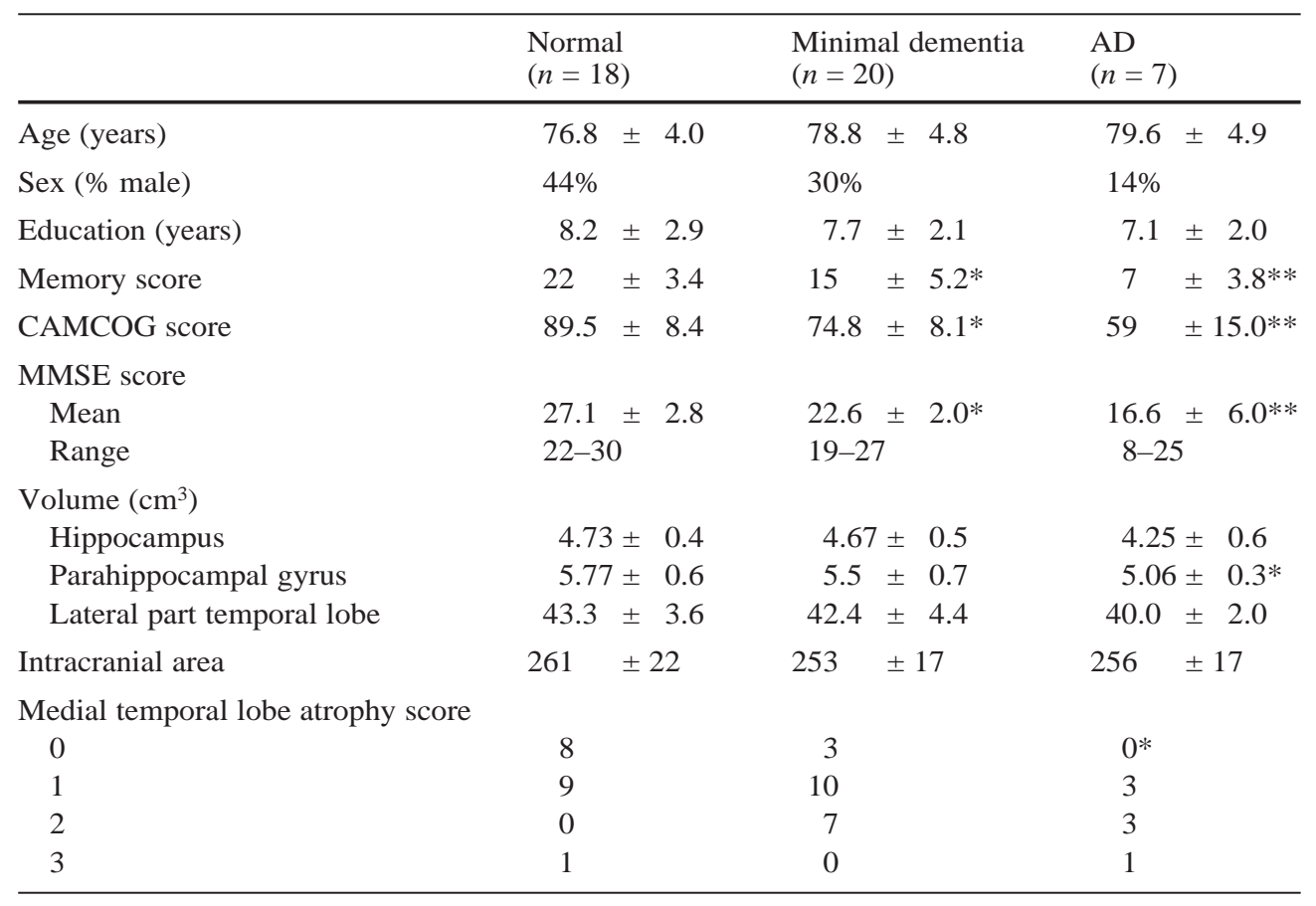


Table 2 Demographic characteristics, cognitive scores, and brain volumes at baseline according to outcome at follow-up

\begin{tabular}{|c|c|c|c|c|c|c|}
\hline & \multirow{2}{*}{$\begin{array}{l}\text { Normal: no demen- } \\
\text { tia at follow-up } \\
\text { (group } 1, n=14 \text { ) }\end{array}$} & \multicolumn{2}{|c|}{ Minimal dementia at baseline } & \multirow{2}{*}{$\begin{array}{l}\text { AD at baseline } \\
\text { (group } 3, n=7 \text { ) }\end{array}$} & \multicolumn{2}{|l|}{$P$} \\
\hline & & $\begin{array}{l}\text { No dementia } \\
\text { at follow-up } \\
\text { (group } 2 \mathrm{a}, n=4)\end{array}$ & $\begin{array}{l}\text { AD at follow-up } \\
\text { (group } 2 \mathrm{~b}, n=9 \text { ) }\end{array}$ & & $\begin{array}{l}\text { Group } \\
2 \mathrm{a} \text { vs } 2 \mathrm{~b}\end{array}$ & $\begin{array}{l}\text { Group } \\
2 \text { b vs } 3\end{array}$ \\
\hline Age (years) & $76.1 \pm 4.8$ & $77.8 \pm 7$ & $79.2 \pm 3.7$ & $79.6 \pm 4.9$ & 0.88 & 0.71 \\
\hline Sex (\% male $)$ & $50 \%$ & $0 \%$ & $33 \%$ & $14 \%$ & 0.21 & 0.38 \\
\hline Education (years) & $8.9 \pm 2.8$ & $8.0 \pm 2.3$ & $7.1 \pm 2.3$ & $7.1 \pm 2.0$ & 0.41 & 0.95 \\
\hline Memory score & $23.1 \pm 1.8$ & $18.3 \pm 4.4$ & $15.1 \pm 5.1$ & $7.0 \pm 3.8$ & 0.31 & 0.005 \\
\hline CAMCOG score & $92.6 \pm 4.3$ & $73.5 \pm 7.3$ & $75.8 \pm 7.6$ & $59.0 \pm 15.0$ & 0.64 & 0.01 \\
\hline $\begin{array}{l}\text { MMSE score } \\
\text { Mean } \\
\text { Range }\end{array}$ & $\begin{array}{l}27.6 \pm 2.3 \\
22-30\end{array}$ & $\begin{array}{l}21.0 \pm 1.8 \\
19-23\end{array}$ & $\begin{array}{l}23.1 \pm 1.2 \\
21-27\end{array}$ & $\begin{array}{l}16.6 \pm 6.0 \\
8-25\end{array}$ & 0.05 & 0.04 \\
\hline $\begin{array}{l}\text { Volume }\left(\mathrm{cm}^{3}\right) \\
\text { Hippocampus } \\
\text { Parahippocampal gyrus } \\
\text { Lateral part temporal lobe }\end{array}$ & $\begin{array}{r}4.83 \pm 0.4 \\
5.92 \pm 0.5 \\
43.2 \pm 3.3\end{array}$ & $\begin{array}{r}5.0 \pm 0.6 \\
5.92 \pm 0.4 \\
43.6 \pm 6.2\end{array}$ & $\begin{array}{r}4.45 \pm 0.4 \\
5.2 \pm 0.7 \\
42.3 \pm 4.2\end{array}$ & $\begin{array}{r}4.25 \pm 0.6 \\
5.06 \pm 0.3 \\
40.0 \pm 2.0\end{array}$ & $\begin{array}{l}0.09 \\
0.04 \\
0.76\end{array}$ & $\begin{array}{l}0.27 \\
0.67 \\
0.49\end{array}$ \\
\hline Intracranial area & \pm 21 & $244 \pm 8$ & \pm 20 & \pm 17 & 0.88 & 0.56 \\
\hline $\begin{array}{l}\text { Medial temporal lobe atrophy score } \\
0 \\
1 \\
2 \\
3\end{array}$ & $\begin{array}{l}6 \\
8 \\
0 \\
0\end{array}$ & $\begin{array}{l}1 \\
2 \\
1 \\
0\end{array}$ & $\begin{array}{l}0 \\
5 \\
4 \\
0\end{array}$ & $\begin{array}{l}0 \\
3 \\
3 \\
1\end{array}$ & 0.24 & 0.39 \\
\hline
\end{tabular}

Fig. 2 Volume of parahippocampal gyrus at baseline according to clinical outcome

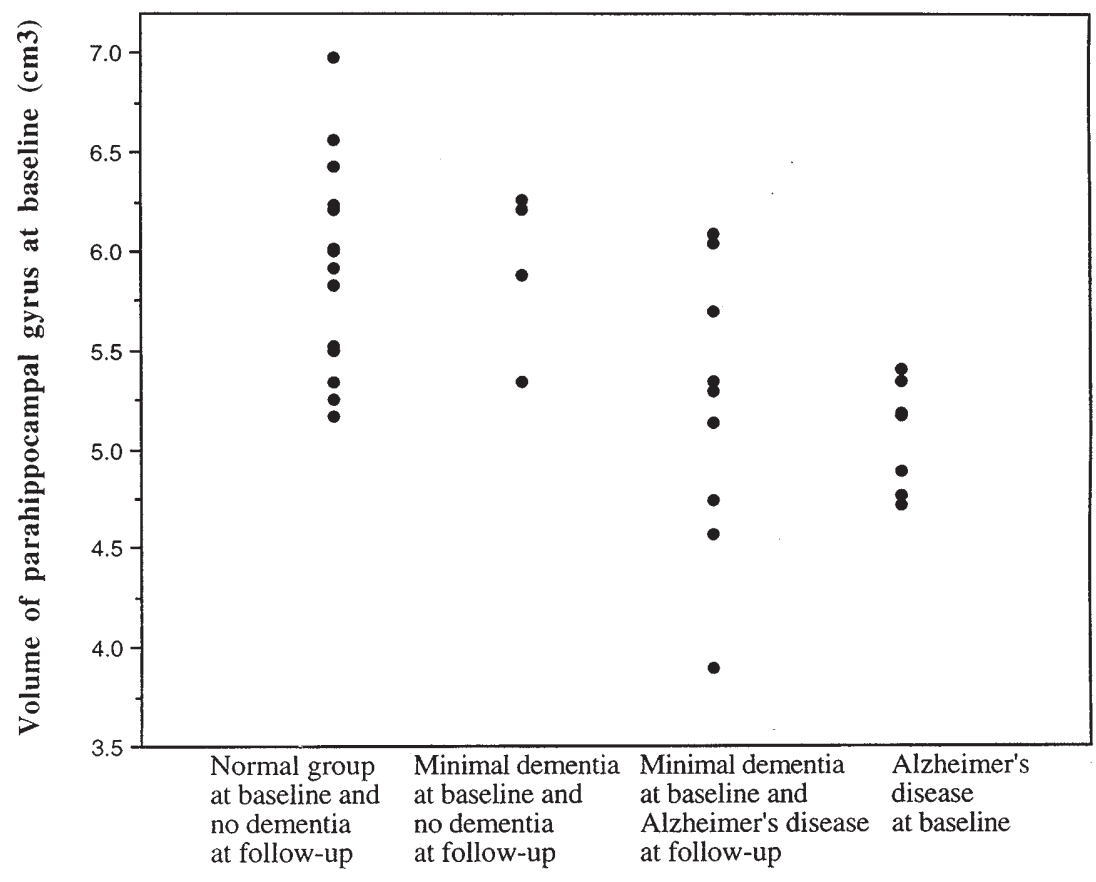

of refusal $(n=7)$, inability to contact $(n=3)$, and death $(n=1)$. Four of these were from the normal group and had a statistical significantly lower CAMCOG total score and a smaller parahippocampal gyrus volume than the other normal subjects. One had severe medial temporal lobe atrophy. This subject was diagnosed as being minimally demented at the first and second follow-up assessments but was then lost to follow-up. Seven subjects from the minimal dementia group who dropped out before the first assessment had baseline characteristics similar to those of the other subjects with minimal dementia. 
Table 3 Logistic regression analyses of clinical outcome

\begin{tabular}{|c|c|c|c|c|c|}
\hline & $\begin{array}{l}\text { Odds } \\
\text { ratio }\end{array}$ & $95 \% \mathrm{CI}$ & $\begin{array}{l}\text { Change in } \\
-2 L L\end{array}$ & $\begin{array}{l}P \text { : Change } \\
\text {-2LL }\end{array}$ & $\begin{array}{l}\text { Correctly } \\
\text { classified }\end{array}$ \\
\hline \multicolumn{6}{|l|}{ Model with single variable } \\
\hline Memory & 0.68 & $0.50-0.91$ & 11.7 & $<0.001$ & $88 \%$ \\
\hline Parahippocampal gyrus & 0.26 & $0.08-\quad 0.86$ & 7.79 & $<0.01$ & $77 \%$ \\
\hline Hippocampus & 0.21 & $0.05-\quad 0.99$ & 5.30 & 0.02 & $69 \%$ \\
\hline Lateral part temporal lobe & 0.97 & $0.85-\quad 1.11$ & 0.15 & 0.70 & $65 \%$ \\
\hline Medial temporal lobe atrophy score (MTL) & 12.2 & $1.4-105.6$ & 9.5 & $<0.01$ & $77 \%$ \\
\hline \multicolumn{6}{|l|}{ Model with two variables } \\
\hline Memory + parahippocampal gyrus & & & 8.8 & $<0.001$ & $96 \%$ \\
\hline Memory & 0.64 & $0.44-\quad 0.93$ & & & \\
\hline Parahippocampal gyrus & 0.15 & $0.02-\quad 0.98$ & & & \\
\hline Memory + hippocampus & & & 4.2 & 0.05 & $92 \%$ \\
\hline Memory & 0.67 & $0.49-\quad 0.94$ & & & \\
\hline Hippocampus & 0.09 & $0.02-\quad 1.29$ & & & \\
\hline Memory + lateral part temporal lobe & & & 0.72 & 0.40 & $80 \%$ \\
\hline Memory & 0.67 & $0.49-0.92$ & & & \\
\hline Lateral part temporal lobe & 0.92 & $0.76-\quad 1.12$ & & & \\
\hline Memory + Medial temporal lobe atrophy score & & & 4.4 & 0.04 & $96 \%$ \\
\hline Memory & 0.70 & $0.51-0.96$ & & & \\
\hline Medial temporal lobe atrophy score & 8.72 & $0.76-101.5$ & & & \\
\hline
\end{tabular}

Change in $-2 \mathrm{LL}$ is for the models with a single variable the change from maximum $-2 \mathrm{LL}$, and for the models with two variables the change from the model with only memory. Odds ratios are for one unit change in the independent variable

Nine of the 27 subjects who completed the follow-up study met the criteria for AD. All of these were diagnosed at baseline as being minimally demented. When compared with the minimally demented subjects with completed follow-up and no dementia $(n=4)$, the demented subjects had the same or better cognitive scores (MMSE), whereas the parahippocampal gyrus was significantly smaller and the hippocampus tended to be smaller (Table 2, Fig. 2). Subjects who developed AD during follow-up differed from those subjects AD at baseline only with respect to the cognitive measures (Table 2). The minimally demented subjects without dementia at follow-up continued to have cognitive impairment at follow-up.

Correlation between brain volumes and cognitive scores

None of the brain volumes were correlated with any of the cognitive scores at baseline in the nondemented group. The volume of the parahippocampal gyrus at baseline was correlated with the change in the memory score $(r=0.53$, $P=0.004)$. When we corrected for the incomplete followup, the volume of the parahippocampal gyrus at baseline was correlated with the change in the CAMCOG score $(r=0.54, P=0.003)$ and the change in the memory score $(r=0.54, P=0.002)$.

\section{Prediction of AD}

The results of the logistic regression analysis with clinical outcome as dependent variable are summarized in Table 3 . The volume of the hippocampus and the parahippocampal gyrus and the medial temporal lobe atrophy

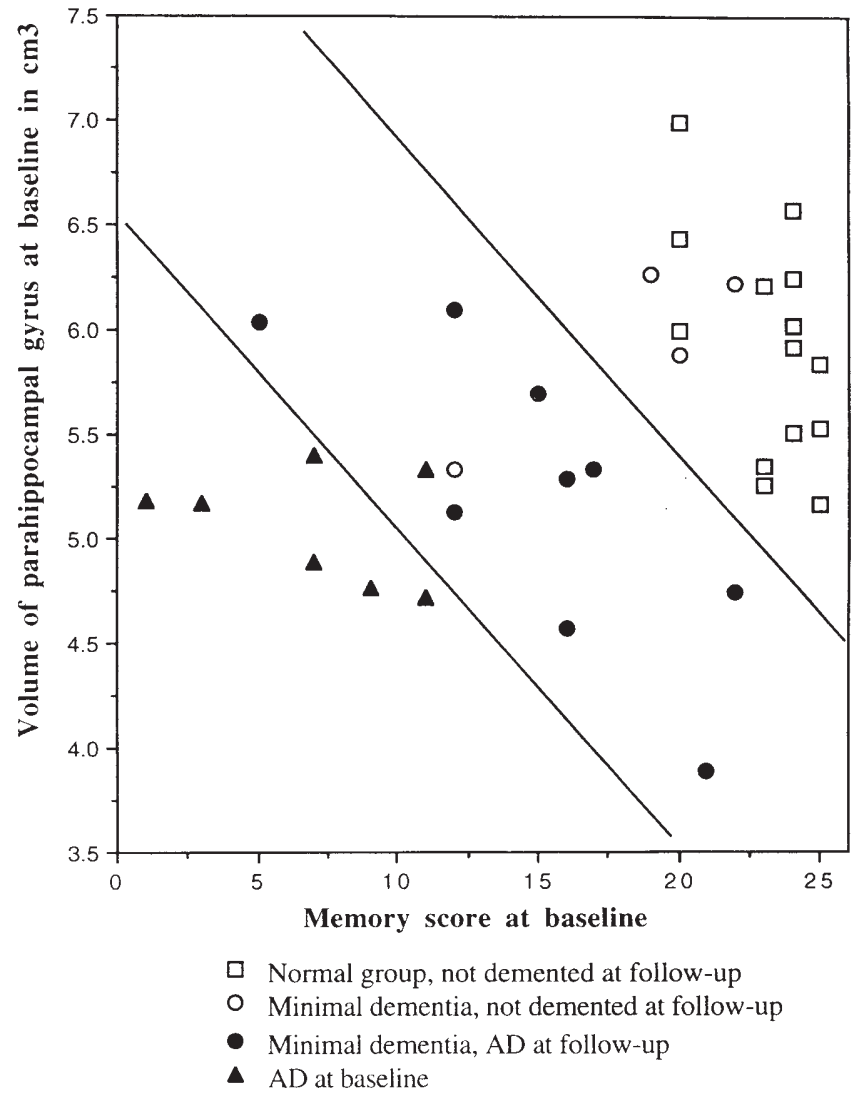

Fig. 3 Relationship between memory score and volume of the parahippocampal gyrus at baseline in subjects with $\mathrm{AD}$ at baseline, with AD at follow-up and no AD at follow-up. Lines, best differentiation of the three groups; open circle in middle part, the subject misclassified as demented by logistic regression 
score increased the accuracy of the model relative to that with the memory score only. The increase was due to the correct classification of subjects with dementia at followup who had at baseline a small volume of the hippocampus or parahippocampal gyrus or a high medial temporal lobe atrophy score, while the memory score was within normal limits. These subjects were false-negatively misclassified as nondemented in the model with only memory score. The best model, which correctly classified most subjects, was the combination of memory score with the volume of the parahippocampal gyrus or the medial temporal lobe atrophy score. Only one subject was misclassified as suffering from AD (see Fig. 3).

\section{Discussion}

We demonstrated that the volume of the parahippocampal gyrus is smaller in subjects with mild cognitive impairment who developed AD within 3 years than in subjects with mild cognitive impairment who do not develop AD. Memory dysfunction is a much better predictor of $\mathrm{AD}$ than the volumes of the hippocampus or the parahippocampal gyrus or the medial temporal lobe atrophy score. The combination of memory function and the volumes of the hippocampus or the parahippocampal gyrus or the medial temporal lobe atrophy score at baseline significantly increased the accuracy of the prediction of the clinical diagnosis of AD compared with that for each measure alone. The increase in accuracy was due to correct classification of subjects who have severe atrophy of the medial temporal lobe but little memory impairment at baseline and who develop AD during follow-up.

The combination of memory function and the volume of the parahippocampal gyrus was slightly superior to the combination of memory function and the volume of the hippocampus, but the small size of our study limits the interpretation of this difference. The memory score plus the medial temporal lobe atrophy score was equal to the combination of memory score plus the volume of the parahippocampus or hippocampus. This makes assessment of the degree of medial temporal atrophy possible in clinical practice because scoring medial temporal lobe atrophy is an easy and reliable method.

Our finding that memory impairment is associated with a higher risk of $\mathrm{AD}$ is consistent with the results of other studies [2, 9, 24, 37], as is the association between atrophy of the medial temporal lobe and preclinical AD $[5,11,17]$. One study compared the combination of psychometrics and a qualitative rating of perihippocampal fluid volume in the prediction of AD in nondemented elderly subjects [5]. Qualitative rating of perihippocampal fluid was found to be superior to the psychometric compound score in predicting AD. The discrepancy between these findings and our own may be the result of the psychometric compound score used, which included non- memory tests, or the different way of assessing medial temporal lobe atrophy.

The volume of the lateral temporal lobe in the subjects with a diagnosis of $\mathrm{AD}$ at follow-up was less reduced than the volumes of the hippocampus and parahippocampal gyrus, which is in agreement with other studies $[3,4,17]$. We found that the volumes of the hippocampus and the parahippocampal gyrus in AD patients is only $10-12 \%$ smaller than that of the normal subjects whereas other authors report differences of $20-50 \%$ [4, 15, 18, 19, 23, 26]. One possible explanation for this difference is that the data from a few normal subjects with severe atrophy and some AD patients with normal volumes affected the overall difference in this small sample. In addition, our scan protocol might not have been sensitive enough in detecting differences in volumes of the hippocampus and parahippocampal gyrus because we measured the volume in only four slices. Another explanation is that our subjects were older (mean age 78 years) than those in the studies (mean 69-76 years), which may have decreased the differences between the groups.

It should be noted that the mildly impaired subjects without dementia continued to have cognitive impairment and may eventually become demented. One may speculate that severe medial temporal lobe atrophy is associated with faster cognitive decline. This is line with the finding that a small volume of the parahippocampal gyrus (our study) or hippocampus at baseline [13] is associated with a decrease in cognitive scores during follow-up. Also the educational level, which tended to be lower in mildly impaired subjects with dementia at follow-up than in the mildly impaired subjects without dementia ( 7.1 vs 8.0 years), may have influenced the rate of decline.

The limitations of this study are the small group size at baseline and the high drop-out rate. The results should therefore be interpreted with care and should be replicated in larger samples. We cannot exclude the possibility that at baseline several of our minimally demented subjects would have been classified as being demented if other dementia criteria had been used [8]. This lack of agreement between dementia criteria, especially in the earliest stages, is a major concern in dementia research and is an incentive to develop new consensus criteria with special interest for very mild dementia. The slice thickness of the MRI may have introduced some measurement error, which might explain why we found no significant differences between minimally demented and normal subjects for all brain measures, and between AD patients and normal subjects for volumes of the hippocampus and lateral part of the temporal lobe volumes despite there being very significant differences in the cognitive scores. The fact that the medial temporal lobe atrophy score did not differ between the minimally demented subjects with and without dementia at follow-up might be explained by the small group size and the use of a $\chi^{2}$ test, which has a low power to detect differences. Nonresponse occurred frequently. In 
selecting subjects from the general population, nonresponse was found to be associated with greater cognitive impairment and poorer physical and mental health [22]. Normal subjects who were lost to follow-up had lower CAMCOG scores and a smaller volume of the parahippocampal gyrus at baseline. Thus, it seems likely that refusal to participate was associated with a higher occurrence of cognitive impairment and probably AD.

In conclusion, our findings indicate that severe medial temporal lobe atrophy is present in some subjects who have mild cognitive impairments at baseline who subsequently develop dementia. The absence of medial tempo- ral lobe atrophy, however, does not exclude the development of dementia. In the majority of the subjects memory impairment was a better predictor for dementia than atrophy of the medial temporal lobe, but the combination of the two increased predictive accuracy. Nondemented subjects with severe atrophy of the medial temporal lobe could be enrolled in drug trials aimed at slowing the progression of $\mathrm{AD}$.

Acknowledgements We thank Huub van der Mortel and Frank Hoogenraad for developing the software, Guillaume Thelissen and Marc Geerlings for technical help, and Menno Witter and Harry Uylings for advise on brain measurements.

\section{References}

1. American Psychiatric Association (1987) Diagnostic and statistical manual of mental disorders IIIR, 3rd revised edn. American Psychiatric Association, Washington

2. Bowen J, Teri L, Kukull W, McCormick W, McCurry S, Larson E (1997) Progression to dementia in patients with isolated memory loss. Lancet 349: 763-765

3. Braak H, Braak E (1992) The human enthorhinal cortex: normal morphology and lamina-specific pathology in various diseases. Neurosci Res 15:6-31

4. Convit A, de Leon M, Tarhish C, et al (1997) Specific hippocampal volume reductions in individuals at risk for Alzheimer's disease. Neurobiol Aging $18: 131-138$

5. de Leon M, George A, Convit A, et al (1993) The radiologic prediction of Alzheimer disease: the atrophic hippocampal formation. AJNR Am J Neuroradiol 14:897-906

6. Derix M, Hofstede A, Teunisse S, et al (1992) CAMDEX-N: de Nederlandse versie van de Cambridge Examination of Mental Disorders of the Elderly. Swets \& Zeitlinger, Lisse

7. Bodick N, Forette F, Hadler D, et al (1997) Protocols to demonstrate slowing of Alzheimer's disease progression. Alzheimer Dis Assoc Disord 11 [Suppl 3]: 50-53

8. Erkinjuntti T, Østbye T, Steenhuis R, Hachinski V (1997) The effect of different diagnostic criteria on the prevalence of dementia. N Engl J Med 337 : 1667-1674

9. Flicker C, Ferris SH, Reisberg B (1991) Mild cognitive impairment in the elderly: predictors of dementia. Neurology 41 : 1006-1009
10. Folstein M, Folstein S, McHugh P (1975) "Mini-Mental State," a practical method for grading the cognitive state of patients for the clinician. J Psychiatr Res 12:189-198

11. Fox N, Warrington E, Freeborough P, et al (1996) Presymptomatic hippocampal atrophy in Alzheimer's disease. Brain 2001-2007

12. Frisoni G, Beltramello A, Weiss C, Geroldi C, Bianchetti A, Trabucchi M (1996) Usefulness of simple measures of temporal lobe atrophy in probable Alzheimer's disease. Dementia $7: 15-$ 22

13. Golomb J, Kluger A, de Leon M, et al (1996) Hippocampal formation size predicts declining memory performance in normal aging. Neurology 47 : 810-813

14. Hughes CP, Berg L, Danziger WL, Coben LA, Martin RL (1982) A new scale for the staging of dementia. $\mathrm{Br}$ J Psychiatry 140:566-572

15. Jack C, Petersen R, Xu Y, et al (1997) Medial temporal lobe atrophy on MRI in normal aging and very mild Alzheimer's disease. Neurology 49:786-794

16. Jack C, Twomey C, Zinsmeister A, Sharbrough F, Petersen R, Cascino G (1989) Anterior temporal lobes and hippocampal formations: normative volumetric measurements from MR images in young adults. Radiology $172: 549-554$

17. Kaye J, Swihart T, Howieson D, et al (1997) Volume loss of the hippocampus and temporal lobe in healthy elderly persons destined to develop dementia. Neurology 48:1297-1304

18. Kesslak J, Nalcioglu O, Cotman C (1991) Quantification of magnetic resonance scans for hippocampal and parahippocampal atrophy in Alzheimer's disease. Neurology $41: 51-54$

19. Laakso M, Soininen H, Partanen K, et al (1995) Volumes of hippocampus, amygdala and frontal lobes in the MRIbased diagnosis of early Alzheimer's disease: correlation with memory functions. J Neural Transm 9:73-86
20. Launer L, Dinkgreve M, Jonker C, Hooijer C, Lindeboom J (1993) Are age and education independent correlates of the Mini-Mental State Exam performance ofcommunity dwelling elderly? J Gerontol 48: 271-277

21. Launer L, Scheltens P, Lindeboom J, Barkhof F, Weinstein H, Jonker C (1995) Medial temporal lobe atrophy in an open population of very old persons. Neurology $45: 747-752$

22. Launer L, Wind A, Deeg D (1994) Nonresponse pattern and bias in a community-based cross-sectional study of cognitive functioning among the elderly. Am J Epidemiol 139:803-812

23. Lehéricy S, Baulac M, Chiras J, et al (1994) Amygdalohippocampal MR volume measurements in the early stages of Alzheimer's disease. AJNR Am J Neuroradiol 15:927-937

24. Linn R, Wolf P, Bachman D, et al (1995) The 'preclinical phase' of probable Alzheimer's disease. Arch Neurol 52:485-490

25. McKhann G, Drachman D, Folstein M, Katzman R, Price D, Stadlan EM (1984) Clinical diagnoses of Alzheimer's disease: report of the NINCDSADRDA Work-Group under the auspices of the Department of Health and Human Services Task Force on Alzheimer's disease. Neurology 34:939-944

26. Pantel J, Schröder J, Schad L, et al (1997) Quantitative magnetic resonance imaging and neuropsychological functions in dementia of the Alzheimer type. Psychol Med 27:221-229

27. Petersen RC, Smith GE, Ivnik RJ, Tangalos EG (1994) Memory function in very early Alzheimer's disease. Neurology $44: 867-872$

28. Reisberg B, Ferris S, De Leon M, Crook T (1982) The global deterioration scale for assessment of primary degenerative dementia. Am J Psychiatry 139:1136-1139 
29. Rombouts S, Machielsen W, Witter M, Barkhof F, Lindeboom J, Scheltens P (1997) Visual association encoding activates the medial temporal lobe: a functional magnetic resonance imaging study. Hippocampus 7:594-601

30. Roth M, Tym E, Mountjoy CQ, et al (1986) A standarized instrument for the diagnoses of mental disorder in the elderly with special reference to the early detection of dementia. Br J Psychiatry 149: 698-709

31. Scheltens P, Launer L, Barkhof F, Weinstein H, van Gool WA (1995) Interobserver reliability of visual assessment of hippocampal atrophy on MRI. J Neurol 242:557-560
32. Scheltens P, Launer L, Weinstein H, Barkhof F, Jonker C (1997) The diagnostic value of MRI and 99mTc HMPAO SPECT for the diagnosis of Alzheimer's disease in a community dwelling elderly population. Alzheimer Dis Assoc Disord 11:63-70

33. Scheltens P, Leys D, Barkhof F, et al (1992) Atrophy of the medial temporal lobes on MRI in Alzheimer's disease and normal ageing: diagnostic value and neuropsychological correlates. J Neurol Neurosurg Psychiatry 55: 967-972

34. Scheltens P, Pasquier F, Weerts J, Barkhof F, Leys D (1997) Qualitative assessment of cerebral atrophy on MRI: inter- and intra-observer reproducibility in dementia and normal aging. Eur Neurol 37:95-99
35. Squire L, Zola-Morgan S (1991) The medial temporal lobe memory system. Science 253: 1380-1386

36. Storandt M, Hill R (1989) Very mild senile dementia of the Alzheimer typeII Psychometric test performance. Arch Neurol 46:383-386

37. Tierney M, Szalai J, Snow W, et al (1996) Prediction of probable Alzheimer's disease in memory impaired patients. Neurology 46:661-665

38. Vermersch P, Scheltens P, Leys D, Barkhof F (1994) Visual rating of hippocampal atrophy: correlation with volumetry. J Neurol Neurosurg Psychiatry $57: 1015$ 